\title{
МЕХАНИЧЕСКИЕ ПРОЦЕССЫ В СИСТЕМЕ С ПОГРУЖНОЙ ЭЛЕКТРОРАЗРЯДНОЙ КАМЕРОЙ. ЭКСПЕРИМЕНТ И МОДЕЛИРОВАНИЕ
}

Использование техпроцессов обработки металлов давлением, основанных на действии электрогидравлического эффекта (ЭГЭ), тормозится из-за методических трудностей анализа всего ансамбля сопутствующих процессов в оборудовании для его изучения, проектирования и оптимизации.

Электрогидравлический эффект представляет собой преобразование импульса электрического тока в жидкости в ее движение. В результате электрического пробоя жидкости образуется канал, заполненный ионизированным паром, через который течет импульсный ток. Выделяющееся тепло омических потерь приводит к росту давления и интенсивному расширению парогазовой полости (ПГП), вследствие чего в окружающей жидкости проявляются три механических фактора: волна давления, расходящиеся потоки жидкости, а также импульсное быстроменяющееся поле давлений. На основе ЭГЭ предложены и разработаны многочисленные технологии и системы, служащие для деформирования или разрушения объектов [1].

В настоящее время подобные гетерогенные механические системы исследуются в рамках направления прикладной механики FSI (Fluid-Structure Interaction), основным методом которого является компьютерное моделирование, основанное на численных решениях нестационарных контактных задач механики сплошных сред [2].

В работах сотрудников Института импульсных процессов и технологий НАН Украины разработана и использована осесимметричная эйлерова вычислительная конечноразностная модель ЭГЭ [3]. С ее помощью выполнен большой объем теоретических исследований, в частности, рассмотрены задачи свободного деформирования цилиндрических длинных упругих и упругопластических оболочек без учета разгрузки.

В работе В. С. Мамутова [4] использована осесимметричная лагранжева конечноэлементная вычислительная модель деформирования упругопластической плоской оболочки. По существу действие ПГП на жидкость заменяется движением жесткого поршня. В работах по FSI отмечено, что применение лагранжевых сеточных вычислительных моделей ограничено из-за разрушения сетки, играющей роль координат материальной системы отсчета, вихрями, присущими движениям жидкости в ограниченном объеме.

В работе Гимадеева [5] методом конформных отображений показано, что для волны давления в жидкости от линейного конечного источника характерно образование фронта в виде эллипсоида вращения.

Все вышеупомянутые осесимметричные модели имеют ограниченное применение, так как в действительности первоначально осесимметричный характер движения (жидкости) ломается при встрече с границами оснастки и объекта, имеющими, в общем случае, несимметричную форму.

В работе [6] описана и изучена трехмерная вычислительная модель ЭГЭ в жесткой неосесимметричной камере. Использован метод ALE [7, 8], малочувствительный к вихрям в жидкости, позволяющий описать взаимодействие жидкости и газа, с одной стороны, и твердого тела, с другой, при высокой степени деформации. Объемная деформация ПГП в расчетах достигала 800 единиц без нарушения устойчивости вычислительного процесса. Показана высокая степень точности асимптотических значений давления и объема ПГП и жидкости, фронт волны давления до встречи с границами камеры имеет предсказанную в [5] форму, обоснована адекватность модели. 
Целью статьи является повышение эффективности математического моделирования системы процессов, реализуемых в технологических устройствах для электрогидравлической штамповки. Задачи статьи: представление пространственной модели гетерогенной системы с погружной камерой, основанной на модели ЭГЭ [6], анализ ее адекватности посредством сравнения расчетных результатов с результатами экспериментов, раскрытие особенностей движения элементов такой системы.

На рис. 1 показана схема экспериментальной установки, включающей наряду с электрофизической (делитель напряжений 11, разрядник 13) и механической (заготовка 1, 2, 4 вытяжное и прижимное кольца, камера 3, погружная разрядная камера 5) системами еще и подсистему регистрации движения элементов (камера СФР 6, импульсные лампы 7, экран 8, осциллограф 9 и 12, пояс Роговского 10, делитель напряжений 11).

На рис. 2 показана схема механической подсистемы (корпусы камеры и погружной камеры, а также расширение области решения условно не показаны). Диаметр заготовки 320 мм, диаметр проходного отверстия вытяжного кольца 252 мм, толщина заготовки 1 мм.

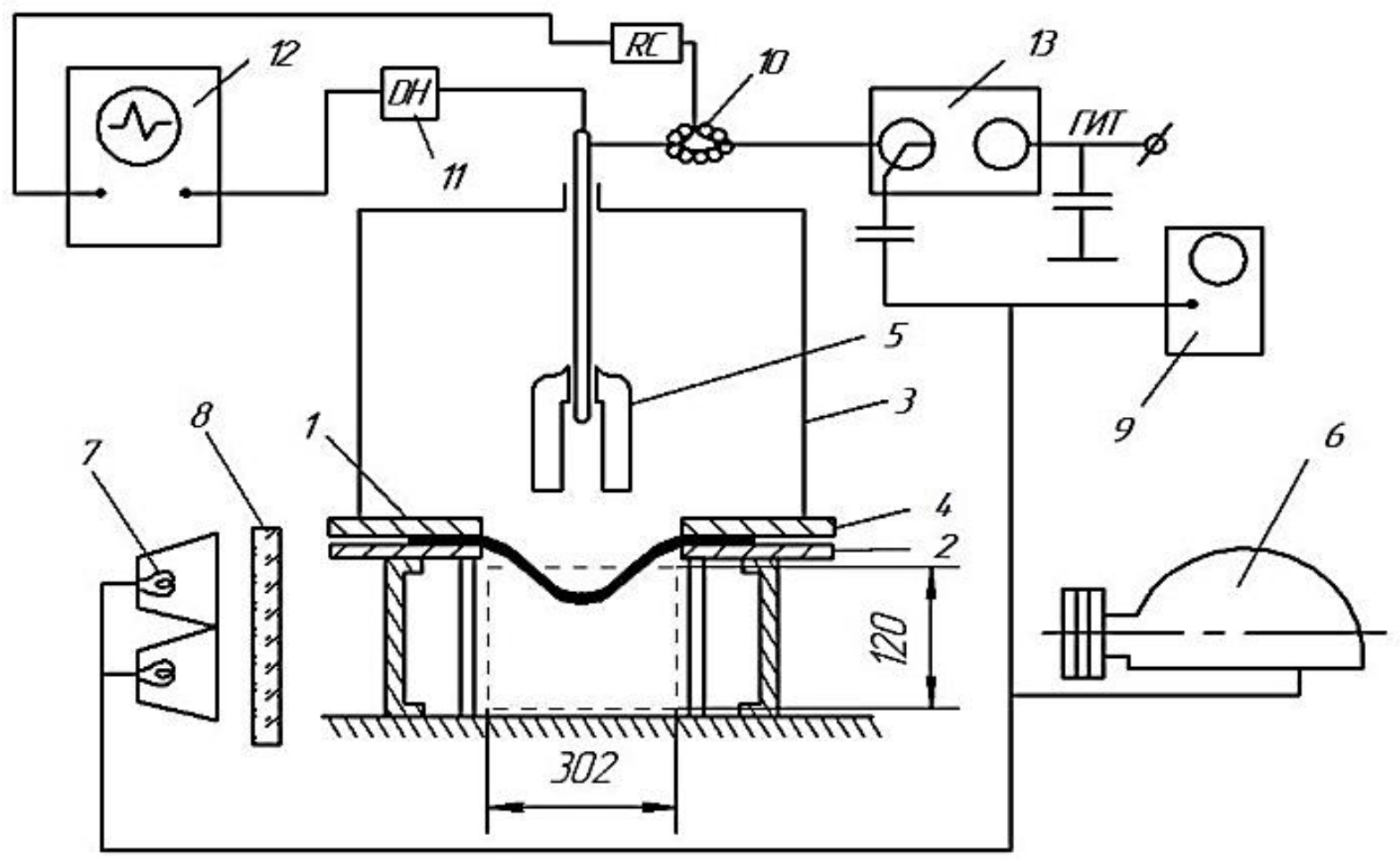

Рис. 1. Схема экспериментальной установки

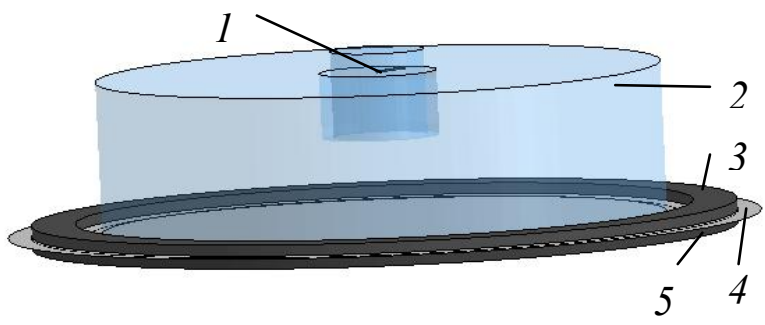

Рис. 2. Схема модели

Материал заготовки - упруго-пластический алюминиевый сплав со степенным деформационным упрочнением вида, $\sigma_{T}=A \times e^{n}$, где $A=4,63 \cdot 10^{8}$ Па, $n=0,122$ коэффициенты упрочнения, начальное значение предела текучести $210^{8}$ Па, плотность 2700 кг/м³. Высота жидкости в камере 70 мм, в погружной камере - 80 мм, диаметр погружной камеры 40 мм. 
Жидкость (вода) плотностью 1000 кг/м³ , объемным модулем сжатия 2,25 $10^{10}$ Па, давлением кавитации 0,1 атм. Среда ПГП - идеальный газ с показателем адиабаты 0,26, плотностью при

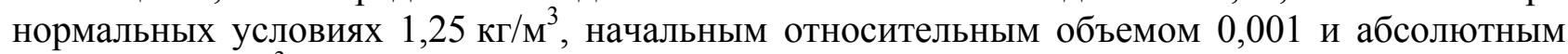
объемом 7,1 мм³

Характеристики электрофизической подсистемы - начальное напряжение конденсаторной батареи 13,5 кВ, емкость разрядной цепи 10 мкФ, индуктивность цепи 1 мкГн, среднее сопротивление канала разряда 0,02 Ом, начальное сопротивление цепи 0,1 Ом. Расчетная зависимость потока тепла в канал разряда показана на рис. 3 красной линией, черная линия представляет аппроксимацию, принятую при решении механической задачи. Запасенная в разрядной системе энергия 9,1 кДж, выделенное в канале разряда тепло 1,7 кДж.

На рис. 4 показаны картины движения фронта волны давления на выходе из канала погружной камеры в последовательные моменты времени (слева направо). Расчетное поле (вверху) показано в виде изобар. Как в расчете (показано синим цветом), так и в эксперименте (граница темного пятна на светлом фоне) наблюдается несимметрия фронта, обусловленная несимметричностью положения канала разряда (в данном случае- левее оси симметрии камеры). В картинах, построенных по результатам расчета, видно, что на выходе из канала фронт и изобары за фронтом принимают уплощенную форму, а после выхода в окружающую жидкость - сферическую форму, что отмечалось в экспериментах. Поле давлений за фронтом имеет слоисто-неоднородную структуру и в расчете, и в эксперименте. Расчетная скорость движения фронта в погружной камере и при выходе из нее превосходит скорость звука и достигает величины 1900 м/с.

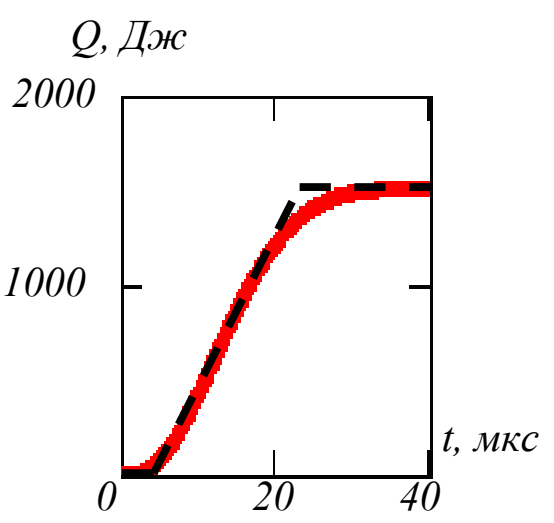

Рис. 3. Зависимость потока тепла в ПГП от времени

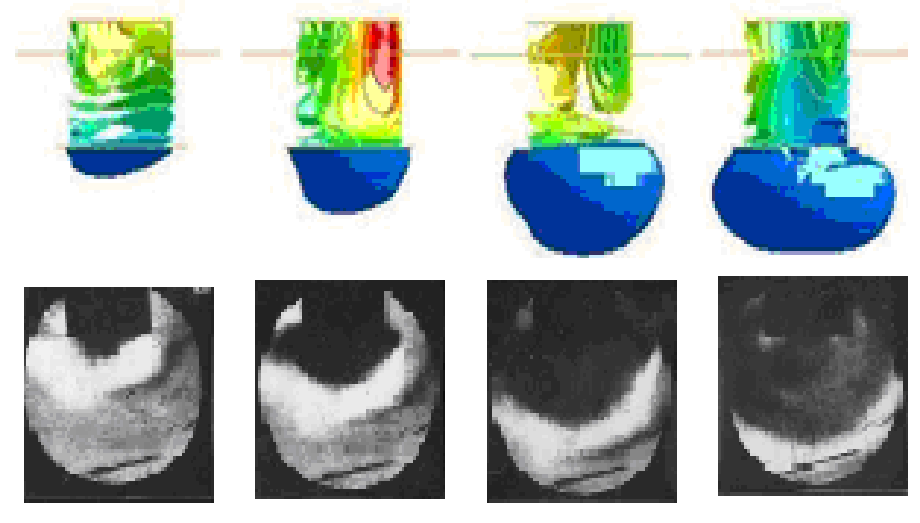

Рис. 4. Движение фронта волны давления на выходе из погружной камеры (вверху расчет, внизу - СФР-грамма)

На рис. 5 показаны теневые картины деформирования заготовки. В верхнем ряду показаны результаты моделирования, зеленым цветом показана поверхность ПГП, коричневым - сеточная модель оболочки; в нижнем ряду показаны соответствующие СФР-граммы. Рис. 5, а соответствует моменту прихода волны давления к заготовке и началу ее деформирования; ПГП, имевшая первоначально форму параллелепипеда с отношением сторон $1: 33$, вытянутого вдоль радиуса камеры, пробрела шаровую форму. На рис. 5, б ПГП практически заполнила погружную камеру, вытеснив жидкость; оболочка принимает коническую форму, периферическая ее часть практически неподвижна. На рис. 5, в ПГП вышла за пределы погружной камеры и приняла округлую форму; изгибная деформация оболочки достигла перетяжного кольца, купол оболочки имеет сферическую форму. На последнем рисунке ПГП расширяется в камере, вытесняя жидкость на периферию; степень объемной деформации ПГП превосходит $1,1210^{5}$. Образующая оболочки имеет две точки перегиба, что говорит о 
переменчивости причин ее деформирования. ПГП расширяется в радиальном направлении, в основном, путем увеличения объема камеры при деформировании оболочки, создавая в жидкости радиальные потоки, превращающиеся в замкнутом объеме камеры в вихри. Деформация оболочки менее заметна, чем фронт волны давления (рис. 4) и ПГП реагирует на начально несимметричное положение канала разряда в погружной камере.

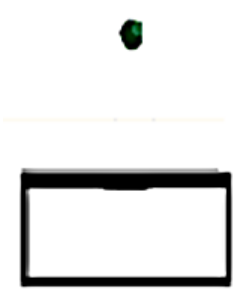

$a$

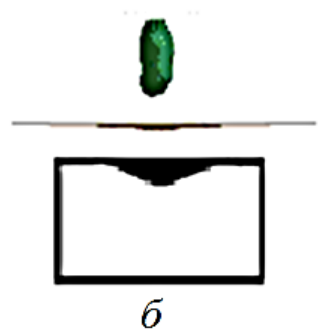

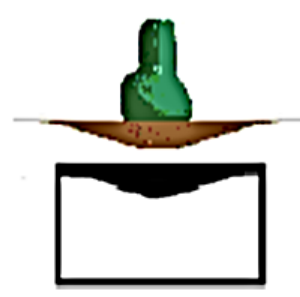

$B$

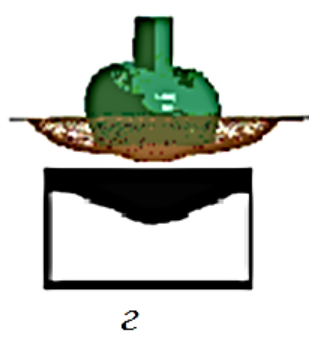

2

Рис. 5. Теневые картины перемещений заготовки (вверху - результаты моделирования, внизу - СФР-граммы)

На рис. 6 показаны расчетные осевые перемещение (вверху) и скорость (внизу) купола оболочки (рис. 5) в зависимости от времени. На графиках вначале значения неизменны, задержка равна времени прохождения волны давления от ПГП до оболочки. Зависимость перемещений имеет вид гладкой функции, что наблюдается и в эксперименте, но экспериментальные перемещения регистрируются непосредственно, в то время как показанные на рис. 6 определены интегрированием расчетных скоростей перемещений. Максимальное перемещение величиной 36 мм достигается в момент времени 1 мс, затем наблюдается уменьшение, обусловленное упругой разгрузкой (пружинением) материала. Деформирование оболочки происходит, в общем, после завершения волновых процессов в системе, следовательно, не эти процессы являются основным фактором деформирования. На графике скорости вначале наблюдается скачок, объясняемый действием волны давления; за короткое время скорость купола достигает 40 \% максимальной скорости. Затем наблюдаются легкие осцилляции, особенно заметные после остановки оболочки, обусловленные упругой реакцией материала оболочки. Малая величина осцилляций говорит о том, что происходит монотонное пластическое деформирование оболочки. В расчетах подобных оболочечных систем без учета жидкости осцилляции значительно больше, что говорит о существенном положительном направляющем (демпфирующем) влиянии жидкости на процесс пластического деформирования оболочки. Неучет жидкости в модели приводит к ее неадекватности.
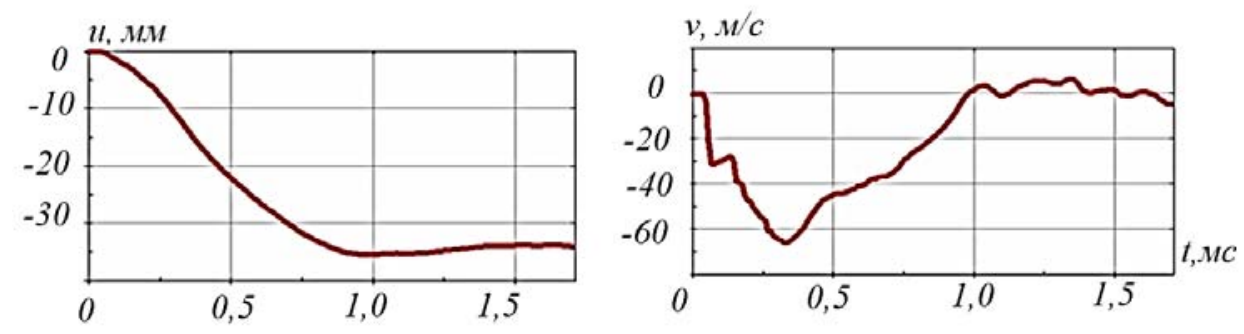

Рис. 6. Зависимость перемещений и скорости перемещений от времени

На рис. 7 показана зависимость от времени давления в верхней части погружной камеры, занимаемой ПГП. Давление растет, пока в ПГП выделяется тепло, максимальное значение давления $111^{7}$ Па. Наблюдается второй пик давления, обусловленный отраженной от оболочки волной давления, его величина меньше на $10 \%$, затем осцилляции, отражающие бурный процесс перемешивания совместно с переотражением волн, на фоне уменьшения давления, обусловленного выходом возмущения (ПГП) за пределы погружной камеры. 


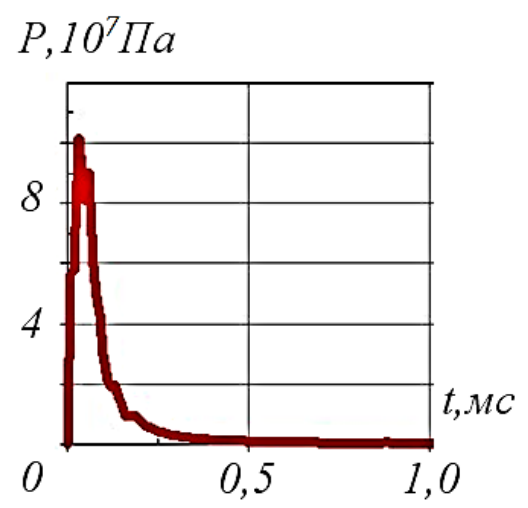

Рис. 7. Зависимость давления в ПГП

На рис. 8 показана зависимость от времени давления в пяти точках, первоначально расположенных на дне камеры на поверхности оболочки (красная линия в центре, зеленая на расстоянии 20 мм, синяя - 50 мм, голубая - 80 мм, лиловая - 120 мм от центра). На каждой линии наблюдается первый пик, обусловленный приходом первой волны давления, задержка роста связана с конечным временем прохождения волны давления; максимальные значения в 17 раз меньше максимальных значений давления в погружной камере (рис. 7). 3атем наблюдается этап импульсного роста давления, который объясняется расширением ПГП за пределы погружной камеры. В центральных точках наибольшее давление наблюдается в начале периода, в периферических - в конце. Отмеченная особенность развития давления приводит к показанной на рис. 5, г особенности деформирования профиля оболочки с двумя точками перегиба, одна из которых лежит на ребре перетяжного кольца, а вторая ближе к центру в зоне между первым и вторым максимумами давления.

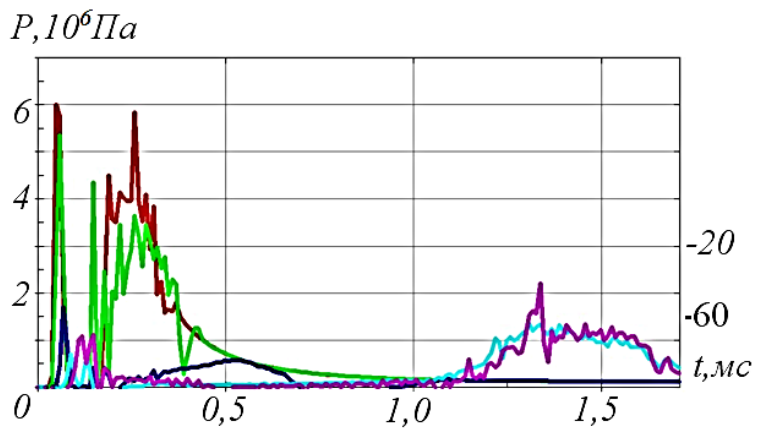

Рис. 8. Зависимость давления на дне камеры

Зоны повторного роста (импульса) давления значительно более продолжительны, чем первичные, обусловленные волной давления. Следует отметить, что в зонах повторного роста заметно влияние волновых эффектов, в особенности для центральных точек. Сравнение рис. 8 и рис. 5 позволяет сделать заключение, что увеличение скорости перемещения купола совпадает по времени с зоной повторного роста давлений в центральных точках.

На рис. 9 показана зависимость пластических деформаций в центре оболочки. Сравнение с рис. 8 (красная линия) дает основание утверждать, что пластические деформации возникают и развиваются во время действия импульсного давления, а затем сохраняют свои значения.

На рис. 10 отражен энергообмен в оболочке (красная линия- полная энергия, зеленаявнутренняя энергия, синяя- кинетическая энергия). Внутренняя энергия равна сумме работ пластической и упругой деформаций; последняя возвращается в систему после 1,5 мс, чем объясняется снижение кривой и приобретение окончательной формы оболочкой. 


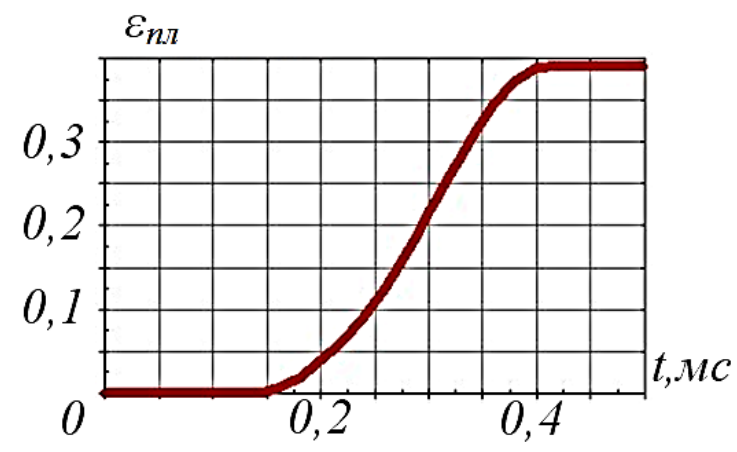

Рис. 9. Зависимость пластических деформаций

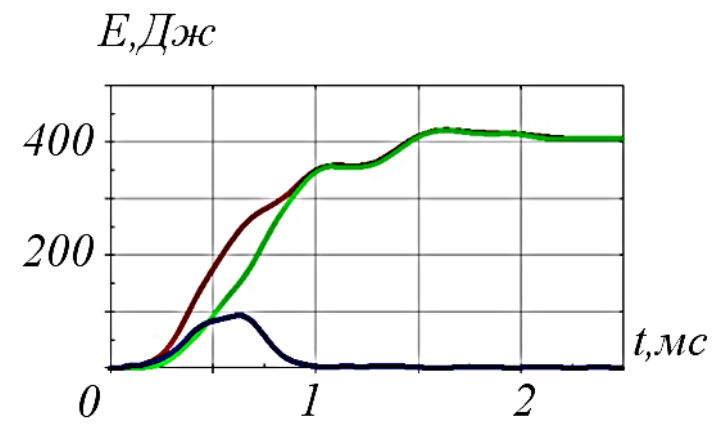

Рис. 10. Зависимость энергий в оболочке

Остаточное значение внутренней энергии, равное 401 Дж, есть работа пластической деформации. Накопление внутренней энергии происходит в два этапа, на первом происходит пластическая деформация центральной части, сопровождающаяся как расширением области пластической деформации, так и ростом уровня пластических деформаций (см. рис. 9). На втором этапе происходит деформирование оболочки на периферии (на ребре перетяжного кольца), соответствующее изменение гораздо меньше, чем на первом этапе. Кинетическая энергия имеет заметное значение на начальном этапе процесса, затем скорости точек оболочки падают (см. рис. 6).

\section{ВЫВОДЫ}

Предложенная вычислительная модель адекватно выражает особенности процессов, выявленные в эксперименте. Наблюдается самопроизвольная смена волновых процессов затухающими колебательными с выходом на стационарное состояние. Величины кинематических, энергетических параметров, а также напряжений и деформаций соответствуют наблюдаемым в экспериментах, а также аналитическим оценкам (скорость движения фронта волны давления).

Основной фактор образования пластических деформаций оболочки в рассмотренной системе - импульс давления, вызванный расширением ПГП. Волна давления, формирующаяся в начале процесса, не приводит к значительным перемещениям и пластическим деформациям; она вызывает скачок скорости деформации, которая снижается тотчас после отражения волны еще упругой оболочкой. Лишь повторный рост (импульса) давления поддерживает рост скорости, собственно перемещений и пластических деформаций оболочки. Подвижная жидкость играет роль меняющего форму пуансона, приспосабливающегося к актуальной форме оболочки. Значение потока жидкости заключается в смачивании поверхности оболочки для восприятия ею импульса давления; поток не является значительным фактором деформирования оболочки.

Влияние импульса давления на пластическое деформирование оболочки кратковременно и локально. С учетом конечности электрической мощности и коэффициента преобразования ее в работу пластического деформирования возможности отдельной камеры ограничены; в системах со свободной поверхностью жидкости (незамкнутых) коэффициент использования падает из-за потерь энергии на свободное движение жидкости. Деформации оболочки растут за счет давления, создаваемого в ПГП, они же приводят к увеличению актуального объема камеры и ПГП, а, следовательно, - снижению давления вплоть до прекращения деформирования оболочки. Без учета податливости оболочки в составе гетерогенной системы модель неадекватна.

Для обширного деформирования оболочки рационально применение несколько камер, в определенном порядке инициирующих импульсы давления [9]. При таком подходе может быть повышена роль волны давления, поскольку она будет догружать оболочку, находящуюся в пластическом состоянии. 


\section{СПИСОК ИСПОЛЬЗОВАННОЙ ЛИТЕРАТУРЫ}

1. Юткин Л. А. Электрогидравлический эффект и его применение в промышленности. Ленинград : Машиностроение, 1986. 253 с.

2. Кривцов В. С., Нарыжный А. Г. Механико-математическое моделирование технологических систем. Технологические системы. 2012. 4(61). С. 51-60.

3. Косенков В. М. Влияние длины канала высоковольтного разряда в воде на эффективность пластического деформирования цилиндрической оболочки. Журнал технической физики. 2011. 81. 10. С. $133-139$.

4. Mamutov A. V., Mamutov V. S. Computer simulation of electrohydraulic sheet metal forming. Nanodesign, technology and computer simulation: proc. of SPIE. v. 659, 65970Y. 2007. pp. 1-5.

5. Гимадеев М. М. Совершенствование методики расчета энергетических параметров ударной волны при высоковольтном электрическом разряде в воде на основе теплофизического подхода: дис. канд. техн. наук: 01.04.14. Набережные Челны, 2009.

6. Нарыжный А. Г. Вычислительная модель электрогидравлического эффекта. Открытые информационные и компьютерные интегрированные технологии: сб-к науч. тр. НАКУ «ХАИ». Харьков, 2012.54. C. $112-124$.

7. Donea J., Huerta A., Ponphot J.Ph., Rodrigues-Ferran A. Arbitrary lagrangian-eulerian methods. Encyclopedia of computational mechanics. Vol. 1. Foundamentals. Wiley. 2004, pp. 1-25.

8. LS-DYNA Keyword User's Manual / Livermore: LSTC. 2006, 2130 p.

9. Тараненко М. Е. Электрогидравлическая штамповка: теория, оборудование, техпроцессы. Харьков : ХАИ, 2011. 272 c.

\section{REFERENCES}

1. Yutkin L.A. Electro-hydraulic effect and its application in industry. Leningrad: Mechanical Engineering. 1986, 253 p. (in Russian).

2. Krivtsov V.S., Naryzhny A.G. Mechano-mathematical modeling of technological systems. Technological systems. 2012, 4 (61), pp. 51-60. (in Russian).

3. Kosenkov V.M. The influence of the channel length of a high voltage discharge in water on the efficiency of plastic deformation of a cylindrical shell. Journal of Technical Physics. 2011, 81, 10, pp.133-139. (in Russian).

4. Mamutov A.V., Mamutov V.S. Computer simulation of electrohydraulic sheet metal forming. Nanodesign, technology and computer simulation: proc. of SPIE. 659, 65970Y. 2007, pp. 1-5.

5. Gimadeev M.M. Improving the methodology for calculating the energy parameters of a shock wave during a high-voltage electric discharge in water based on the thermophysical approach. Ph.D Dissertation. 01.04.14. Naberezhnye Chelny. 2009. (in Russian).

6. Naryzhny A.G. Computational model of electro-hydraulic effect. Open information and computer integrated technologies: collection of scientific papers. Kharkov: KhAI. 2012, 54, pp. 112-124. (in Russian).

7. Donea J., Huerta A., Ponphot J.Ph., Rodrigues-Ferran A. Arbitrary Lagrangian-Eulerian Methods. Encyclopedia of computational mechanics. Vol. 1. Foundamentals. Wiley. 2004, pp. 1-25.

8. LS-DYNA Keyword User's Manual / Livermore: LSTC. 2006, 2130 p.

9. Taranenko M.E. Electro-hydraulic stamping: theory, equipment, technological processes. Kharkov: KhAI. 2011, 272 p. (in Russian).

Нарыжный А. Г. - канд. техн. наук, доц. НАКУ «ХАИ»; E-mail: narizniyag@ukr.net;

Тараненко М. Е. - д-р техн. наук, проф., зав. каф. НАКУ «ХАИ»;

E-mail: m.taranenko@khai.edu.

НАКУ «ХАИ» - Национальный аэрокосмический университет им. Н. Е. Жуковского «Харьковский авиационный институт», г. Харьков, Украина. 